\title{
Secuencia de bridas amnióticas y atresia bilateral de coanas: reporte de un caso
}

\author{
Amniotic band sequence and bilateral choanal atresia: a case report
}

\author{
M. en C. Azucena Del Toro-Valero ${ }^{a, c}$, Dr. Alejandro Estrada-De la Fuente ${ }^{b}$, Dr. Héctor Velázquez Santana, \\ Dr. Jorge Glicerio González $z^{b}$ Dra. María Cristina Navarro Meza ${ }^{b}$ Dra. Lilia Ortega-Hinojosa ${ }^{b} y$ \\ Dra. en C. María Guadalupe López-Cardona ${ }^{a, c}$
}

\section{RESUMEN}

La secuencia de bridas amnióticas (SBA) es un grupo de malformaciones de tipo disruptivo que afecta principalmente las extremidades; clínicamente, se observan anillos de constricción y linfedema en dedos, brazos y piernas; pseudosindactilias y acrosindactilias; además, existe amputación congénita de las extremidades por tumefacción distal; en algunos pacientes se han comunicado, con menor frecuencia, alteraciones craneofaciales y del tronco. La etiología es aún desconocida y la mayoría de los casos son aislados. En este informe presentamos el caso de un paciente masculino, de 45 días de nacido, con diagnóstico de SBA y atresia bilateral de coanas como hallazgo adjunto; se revisan las posibles causas delSBA y las alteraciones asociadas. Palabras clave: secuencia de bridas amnióticas, atresia bilateral de coanas, disrupción de extremidades, craneofaciales y del tronco.

\section{SUMMARY}

Amniotic band sequence (ABS) is a group malformation that mainly affects limbs; clinically, constriction rings and lymphedema of the fingers, arms and legs, acrosyndactyly and pseudosyndactyly are observed; also there is congenital amputation of limbs due to distal swelling. Less frequently, craniofacial and trunk involvement are reported in some patients. Etiology is still unknow and most cases are isolated. In this report we present the case of a 45-day-old male with diagnosis of SBA and bilateral choanal atresia as attached finding, and review possible causes of SBA and associated alterations.

Key words: amniotic band sequence, bilateral choanal atresia, limb, craniofacial and trunk disruption.

a. Departamento de Fisiología, Centro Universitario de Ciencias de la Salud, Universidad de Guadalajara. Guadalajara, Jalisco, México.

b. Hospital Regional "Dr. Valentín Gómez Farías", ISSSTE. Zapopan, Jalisco, México.

c. Becario CONACYT.

\section{Correspondencia:}

Dra. en C. María Guadalupe López Cardona:

lopez_cardona_mg@hotmail.com

Conflicto de intereses:

Ninguno que declarar.

Recibido: 9-12-10

Aceptado: 1-6-11

\section{INTRODUCCIÓN}

La secuencia de bandas amnióticas (SBA) es una alteración de tipo disruptivo, ${ }^{1}$ caracterizada principalmente por anillos de constricción, linfedema, amputación de las extremidades fetales y acrosindactilia; además, secundariamente, se han comunicado alteraciones craneofaciales y del tronco. ${ }^{1-4}$ Se la denomina también complejo de ADAM (del inglés, amniotic deformity, adhesions, mutilations); ${ }^{3}$ con una prevalencia de 1/11 200 nacidos vivos en América latina. ${ }^{5}$ Su etiología aún se desconoce y la mayoría de los casos comunicados son aislados. ${ }^{1} \mathrm{La}$ atresia bilateral de coanas (ABC), un estrechamiento $u$ obliteración primario de la apertura nasal posterior, ${ }^{6}$ no ha sido comunicada como parte del SBA.Dentro de las alteraciones en las estructuras fetales, la secuencia es un encadenamiento de eventos relacionados entre sí por un mecanismo de producción en cascada que surgen de una anomalía primaria. Cuatro tipos de defectos pueden causar una secuencia:

1. malformación: anomalías morfológicas de órganos o partes del cuerpo se producen por un proceso de desarrollo anormal;

2. deformación: no existe problema en el embrión, pero fuerzas mecánicas causan un trastorno morfogenético;

3. disrupción: por procesos destructivos (de origen vascular, infeccioso o mecánico) que alteran la estructura fetal después de la formación normal; ${ }^{7} \mathrm{y}$

4. displasia: falta de organización normal de las células de uno o de varios tejidos. ${ }^{1,7}$

El propósito de este informe es presentar un paciente con SBA típico al que se asocia atresia bilateral de coanas, malformación no comunicada anteriormente en esta entidad; además, se revisan las teorías etiológicas del SBA.

\section{CASO CLÍNICO}

Paciente masculino de 45 días de edad, producto de la tercera gestación de madre de 34 y padre de 40 años de edad, no consanguíneos; con 
dos hermanos aparentemente sanos; sin antecedentes familiares de importancia. Durante todo el embarazo la madre cursó con tos productiva, persistente, sin predominio de horario, tratada con nebulizaciones con salbutamol y, en el segundo trimestre de gestación, con infecciones vaginales reiteradas cuyo manejo se desconoce. El producto fue obtenido por cesárea a las 36 semanas de gestación; peso: $2800 \mathrm{~g}$, con atresia bilateral de coanas $(A B C)$, encefalocele, hidrocefalia, microftalmía derecha y restos de bandas de constricción oscuras en dedos de manos y pies; sin datos de las características de la placenta. $\mathrm{Al} 5^{\text {to }}$ día de vida se le colocó válvula de derivación ventriculoperitoneal y se realizó plastia de encefalocele; a las dos semanas desarrolla neumonía nosocomial. A la exploración física actual, peso: $3150 \mathrm{~g}$ (percentil 3); talla: $52 \mathrm{~cm}$ (percentil 20); plagiocefalia frontotemporal izquierda, dolicocefalia, cicatriz de plastia en región occipital de $10 \mathrm{~cm}$ de longitud y válvula de pudens en región temporal derecha, frontal prominente; ausencia de cejas; microftalmía derecha; puente nasal deprimido; hiperplasia gingival; paladar ojival; pectus excavatum; hernia umbilical; marca de constricción circular por bandas en región distal de antebrazo derecho y dedos 2, 3 y 4; además de aplasia ungueal y acrosindactilia de éstos; mano izquierda con marca de constricción circular por bandas en $4^{\text {to }}$ dedo y acrosindactilia con ausencia ungueal de dedos $2^{\text {do }}$ y $3^{\text {ro }}$; pie derecho con acrosindactilia de dedos $2^{\text {do }}$ y $3^{\text {ro }}$; pie izquierdo ausencia de dedos $1^{\text {ro }}, 2^{\text {do }}$ y $3^{\text {ro }}$, acrosindactilia y marca de banda de constricción de $5^{\text {to }}$ dedo (Figura 1 ).

Los estudios de laboratorio se encontraron dentro de parámetros normales; el cariotipo con resolución de 550 bandas y técnica de bandeo $\mathrm{G}$ en 40 metafases se informó: 46, XY; en las radiografías se observó ausencia de la $2^{\text {da }}$ falange distal de mano derecha y de $2^{\text {da }}$ y $3^{\text {era }}$ falanges distales en mano izquierda; pie derecho, pérdida de falanges medias y distales; en pie izquierdo ausencia de todas las falanges distales. Ecocardiograma normal. La tomografía axial computada simple de cráneo reveló: microftalmía derecha, encefalocele frontoparietal y megaventrículo derecho (Figura 2). La ABC con placa atrésica mixta se confirmó por tomografía axial computada, realizándose corrección vía endoscópica a los 10 meses de edad (Figura 3).

FIgURA 1. Fotografías clínicas del paciente. A: frontal prominente, ausencia bilateral de cejas, microftalmía derecha, puente nasal deprimido. B: mano derecha con sindactilia fenestrada (acrosindactilia) y ausencia de uñas de $2^{\text {do }}, 3^{\text {ro }}$ y $4^{\text {to }}$ dedos, y marca de bandas de constricción a nivel distal (flecha negra). C: mano izquierda con marca de bandas de constricción en primer y $4^{\text {to }}$ dedos (flechas negras), sindactilia fenestrada (acrosindactilia) de $2^{\text {do }} y 3^{\text {er }}$ dedos (flecha roja). D: marca de bandas de constricción a nivel distal de pierna derecha (flecha negra) y pie derecho con sindactilia fenestrada (acrosindactilia) de $2^{\text {do }}$ y $3^{\text {er }}$ dedos (flecha roja). E: Pie izquierdo con ausencia de dedos $1^{r o}, 2^{\text {do }}$ y $3^{\text {ro }}$ y sindactilia fenestrada (acrosindactilia); banda de constricción de $5^{\text {to }}$ dedo (flecha negra)

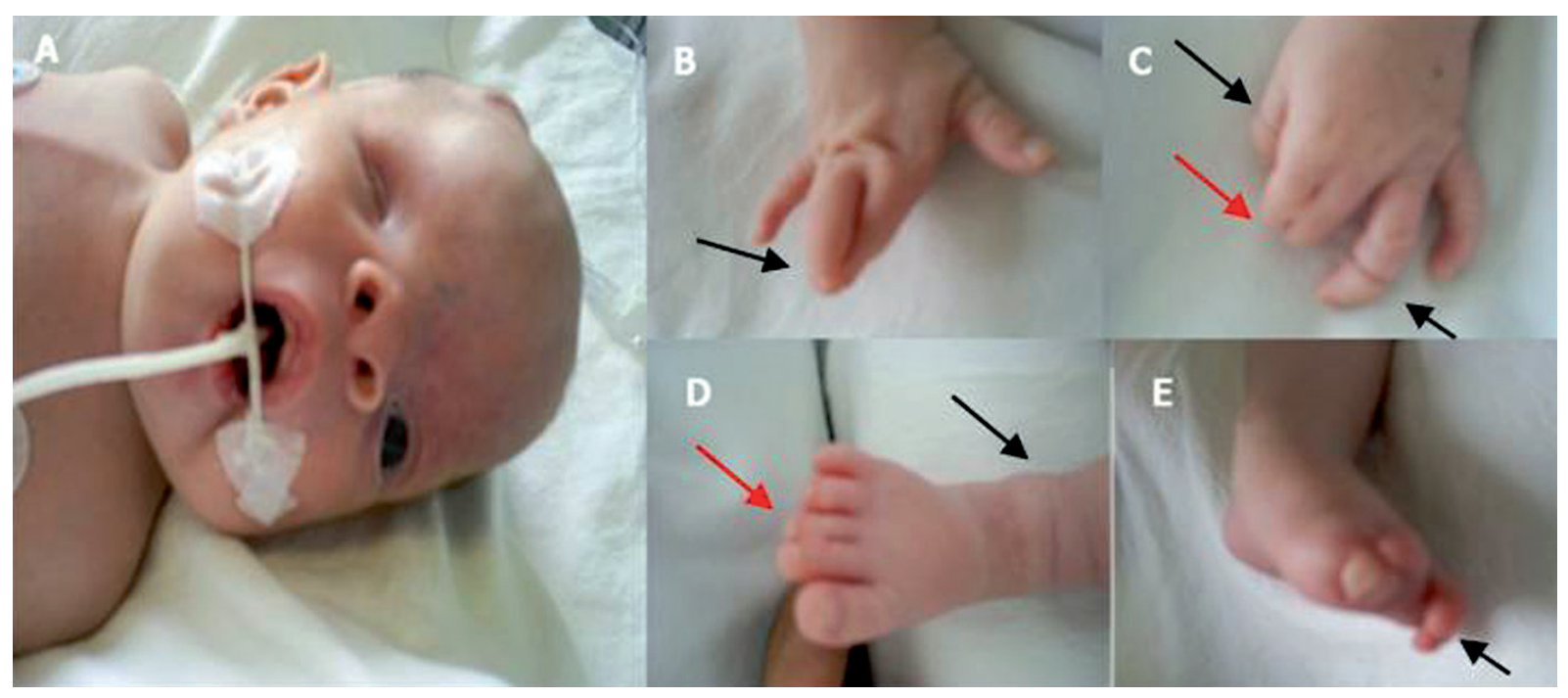




\section{DISCUSIÓN}

La etiología del SBA es aún desconocida y polémica. Existen dos teorías, la endógena propuesta por Streeter en 1930, que supone que ciertos errores del desarrollo en la formación del tejido conectivo de las extremidades causan anomalías ${ }^{8}$ y la fuerza mecánica de las bandas amnióticas compromete la vascularización fetal, alterando la programación y organización básica del feto durante la embriogénesis; ${ }^{1,9}$ por otro lado, la teoría exógena, la más aceptada ${ }^{10}$ y propuesta por Torpin en 1965, describe la ruptura temprana del amnios $^{1,11}$ y existencia de oligohidramnios, con daño de las partes fetales por el contacto del feto con el mesodermo de la cavidad coriónica y desarrollo de bandas fibrosas desde la superficie del amnios fragmentado que atrapan estructuras y causan anillos de constricción en dedos, amputaciones $\mathrm{y}$ abrasiones de la piel que producen los defectos de disrupción. . $^{1,10,11}$ Otras anomalías existentes (como las de órganos internos) no pueden ser explicadas por las teorías anteriores, por lo cual Lockwood ${ }^{12}$ propuso una tercera teoría, llamada vascular, donde los daños vasculares fetales superficiales producen sangrado, ulceraciones y lesiones ectodérmicas con amputaciones posteriores $^{1,10}$ Van Allen y cols. (1992) presentan evidencia de infartos uterinos por émbolos que pueden causar defectos terminales de reducción de extremidades y anillos de constricción similares a los

FIGURA 2. Tomografía axial computarizada simple de cráneo que revela microftalmía derecha (flechas blancas en A y B); encefalocele y megaventrículo derecho (flechas blancas en C y D)

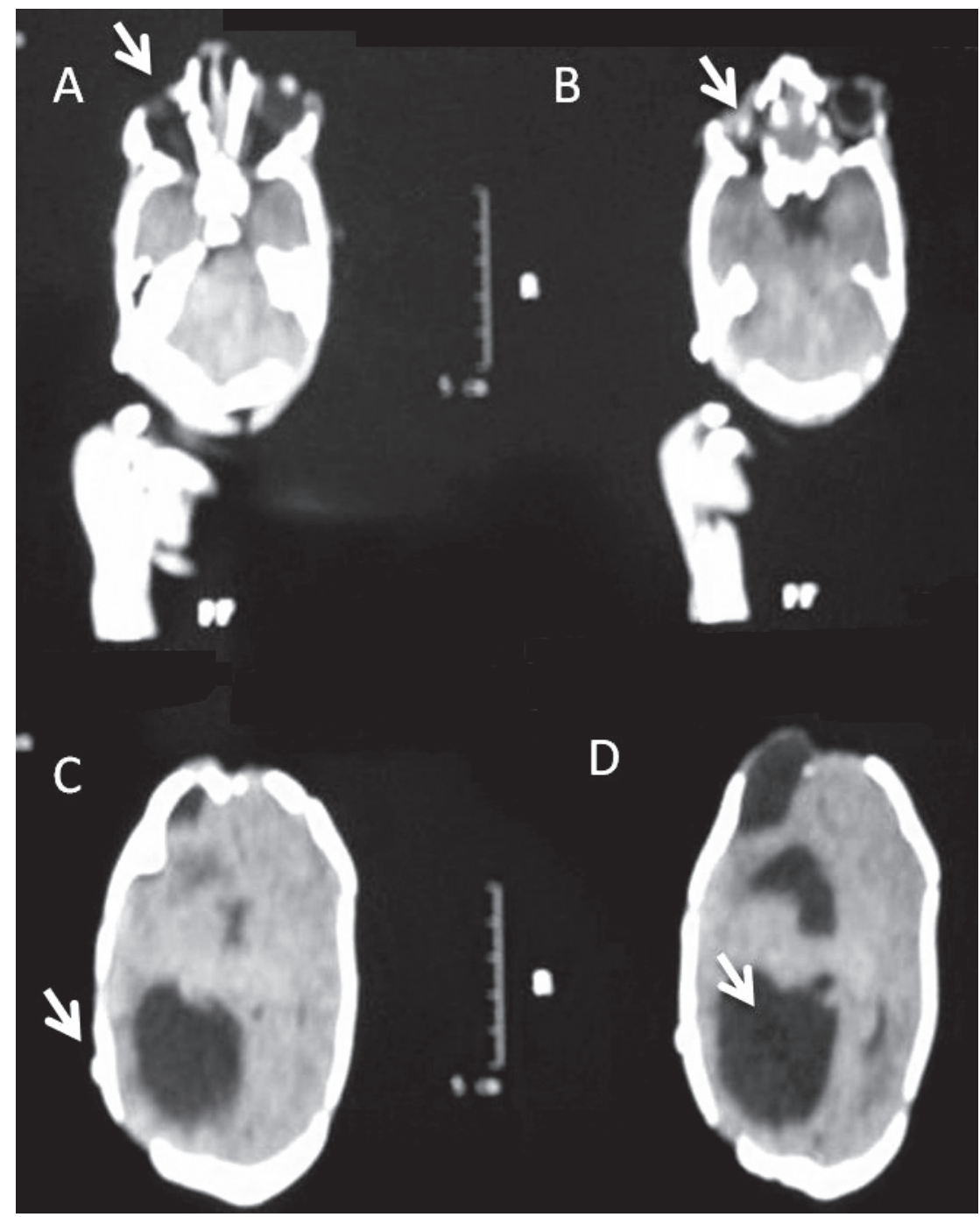


de la SBA en ausencia de anomalías del amnios. ${ }^{13}$ Por lo tanto, con las teorías descritas, se concluye que la SBA puede corresponder a etiologías distintas, no del todo aclaradas hasta nuestros días. ${ }^{12}$ Se trata de un cuadro heterogéneo en cuanto a sus posibles causas. ${ }^{5}$

Las anomalías principales encontradas en la SBA son la amputación de extremidades o dedos por bandas de constricción, linfedema, seudosindactilia y acrosindactilia ${ }^{2-4}$ (cutánea si involucra piel o compleja cuando involucra hueso; completa si involucra uñas e incompleta si no lo hace), ${ }^{14}$ características presentes en la mayoría de los casos. ${ }^{9}$ Sin embargo, el $77 \%$ de los pacientes presenta además alteraciones craneofaciales, torácicas, de columna, abdominales y genitales. ${ }^{2-4}$ Se han comunicado casos con manifestaciones clínicas atípicas, por lo que la expresión clínica es muy variable en la SBA. ${ }^{2,3}$

El diagnóstico diferencial incluye el síndrome de Adams-Oliver, que es una raro cuadro genético con un tipo de herencia tanto autosómico dominante como recesivo; los criterios diagnósticos principales son defectos terminales transversos de extremidades y aplasia cutis congénita. ${ }^{15}$

En cuanto a la atresia de coanas, sus bases etiopatológicas no están claras actualmente. Se pueden explicar por la persistencia de las membranas bucofaríngea y nasobucal del mesodermo, que forma adherencias en la región coanal o por disfunción mesodérmica secundaria a factores locales. ${ }^{6}$ La última teoría podría ser la explicación del caso comunicado en este artículo, ya que la compresión del feto durante la gestación por el oligohidramnios podría ser un factor local, por la compresión de cavidades craneofaciales, que impidiera que no se llevara a cabo el adelgazamiento de la membrana que separa las cavidades nasales de las orales ni la ruptura posterior del epitelio nasal y oral para la formación de las coanas (narinas posteriores). En la bibliografía médica no existen otros estudios que hayan relacionado a la SBA con atresia de coanas bilateral.

Una de las posibles explicaciones de la SBA en nuestro paciente es que la madre tuvo tos y expectoración durante la gestación, por lo que pudo haber un esfuerzo constante al toser que causara el desprendimiento de las bandas y la exposición directa del feto al mesodermo de la cavidad coriónica, lo cual refuerza la teoría exógena de Torpin. Se han comunicado tanto casos aislados como familiares, ${ }^{2,3}$ sin un tipo de transmisión de herencia específico. ${ }^{2}$ El diagnóstico oportuno de la SBA es esencial para brindar atención integral al paciente y asesoramiento genético a la familia. Los casos esporádicos no representan un riesgo aumentado para futuros embarazos de la pareja, ${ }^{2}$ información que es relevante en el asesoramiento genético.

Figura 3. Atresia bilateral de coanas. Visión endoscópica de la porción coanal cuya luz se encuentra disminuida en un $95 \%$ (flechas)

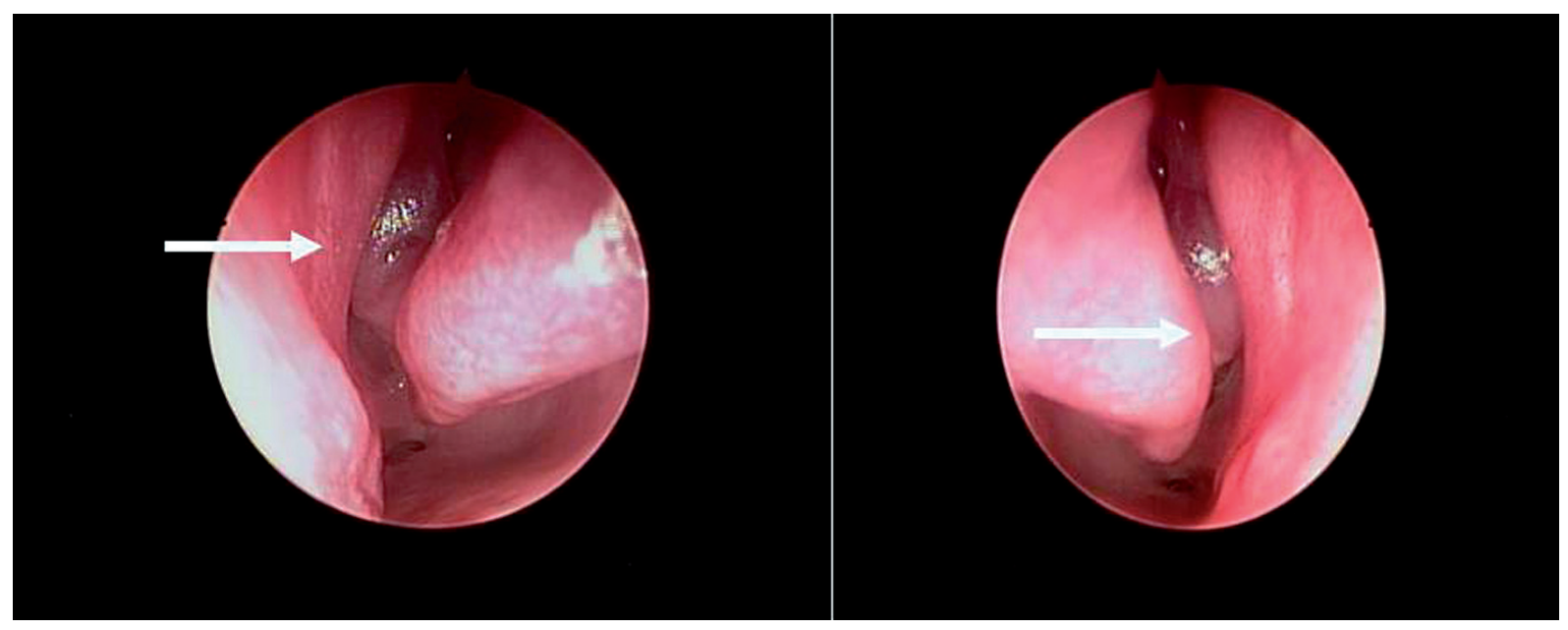




\section{BIBLIOGRAFÍA}

1. Bibas Bonet H, Atar MF, Espíndola Echazú M. Síndrome de bridas amnióticas. Arch Argent Pediatr 2002;100(3):240-44.

2. Lubinsky M, Sujansky E, Sanger W, Salyards P, Severn C. Familial amniotic bands. Am J Med Genet 1983; 14(1):81-7.

3. Keller H, Neuhauser G, Durkin-Stamm MV, Kaveggia EG, et al. 'ADAM complex' (amniotic deformity, adhesions, mutilations)--a pattern of craniofacial and limb defects. Am J Med Genet 1978; 2(2):81-98.

4. Jones KL, Smith DW, Hall BD, Hall JG, et al. A pattern of craniofacial and limb defects secondary to aberrant tissue bands. J Pediat 1974; 84(1):90-5.

5. Orioli IM, Ribeiro MG, Castilla EE. Clinical and epidemiological studies of amniotic deformity, adhesión and mutilation (ADAM) sequence in a South American (ECLAMC) population. Am J Med Genet A 2003; 118A(2):135-45.

6. Burrow TA, Saal HM, de Alarcon A, Martin LJ, et al. Characterization of congenital anomalies in individuals with choanal atresia. Arch Otolaryngol Head Neck Surg 2009; 135(6):543-47.

7. Aviña Fierro JA, Terence Wilson B. Síndrome dismórfico con anomalías congénitas múltiples: Clasificación actual. Rev Mex Pediatr 2009; 76(3):132-5.

8. Schwärzler P, Moscoso G, Senat MV, Carvalho JS, et al. The cobweb syndrome: first trimester sonographic diagnosis of multiple amniotic bands confirmed by fetoscopy and pathological examination. Hum Reprod 1998; 13(10):2966-69.
9. Bamforth JS. Amniotic band sequence: Streeter's hypothesis reexamined. Am J Med Genet 1992; 44(3):280-7.

10. Chatzigeorgiou K, Theodoridis T, Efstratiou I, Athanasiadis A, et al. Strangulation of the umbilical cord by an amnion band- a rare cause of intrauterine demise: a case report. Cases Journal 2009; 2:9108. [Acceso: 31 de agosto de 2010]. Disponible en: http:/ / www.casesjournal.com/ content $/ 2 / 1 / 9108$

11. Martínez-Frías ML. Epidemiological characteristics of amniotic band sequence (ABS) and body wall complex (BWC): Are they two different entities? Am J Med Genet 1997; 73(2):176-179.

12. Lockwood C, Ghidini A, Romero R, Hobbins JC. Amniotic band syndrome: reevaluation of its pathogenesis. Am J Obstet Gynecol 1989; 160(5 Pt1):1030-3.

13. Van Allen MI, Siegel-Bartelt J, Dixon J, Zuker RM, et al. Constriction bands and limb reduction defects in two newborns with fetal ultrasound evidence for vascular disruption. Am J Med Genet 1992; 44(5):598-604.

14. Tonkin MA. Failure of differentiation part I: Syndactyly. Hand Clin 2009; 25(2):171-93.

15. Kalina MA, Kalina-Faska B, Paprocka J, Jamroz E, et al. Do children with Adams-Oliver syndrome require endocrine follow-up?. New information on the phenotype and management. Clin Genet 2010; 78(3):227-35. 